\title{
Performance of a Low-Power Cylindrical Hall Thruster*
}

\author{
Kurt A. Polzin, ${ }^{\dagger}$ Thomas E. Markusic, ${ }^{\ddagger}$ Boris J. Stanojev, ${ }_{2}^{\S}$ and Amado Dehoyos ${ }^{\llbracket}$
}

NASA-Marshall Space Flight Center

Huntsville, AL 35812

\author{
Yevgeny Raitses, ${ }^{* *}$ Artem Smirnov, ${ }^{\dagger \dagger}$ and Nathaniel J. Fisch ${ }^{\ddagger \ddagger}$ \\ Princeton University Plasma Physics Laboratory
}

P.O. Box 451

Princeton, New Jersey 08543

\footnotetext{
*This material is declared a work of the U.S. Government and is not subject to copyright protection in the United States. ${ }^{\dagger}$ Research Scientist. Member AIAA. Corresponding author: kurt.a.polzin@ nasa.gov

${ }^{\ddagger}$ Research Scientist. Member AIAA. Presently: Propulsion Test Manager, Space Exploration Technologies, 1 Rocket Rd., McGregor, TX 76657.

${ }^{\S}$ Research Engineer, Madison Research Corporation. Member AIAA.

'Summer Research Student. Member AIAA. Presently: Test Engineer, Space Exploration Technologies, 1 Rocket Rd., McGregor, TX 76657.

${ }^{* * *}$ Research Physicist. Member AIAA,

${ }^{\dagger}$ Graduate Research Assistant. Member AIAA.

¥¥rofessor, Astrophysical Sciences Dept. Member AIAA.
} 


\section{Nomenclature}

B, $B$ - magnetic induction [T]

$I_{\text {sp }}$ - specific impulse $[\mathrm{s}]$

$\mu_{e}$ - magnetic moment $[\mathrm{J} / \mathrm{T}]$

\section{INTRODUCTION}

Recent mission studies ${ }^{1,2}$ have shown that a Hall thruster which operates at relatively constant thrust efficiency (45-55\%) over a broad power range (300W - 3kW) is enabling for deep space science missions when compared with state-of-the-art ion thrusters. While conventional (annular) Hall thrusters can operate at high thrust efficiency at $\mathrm{kW}$ power levels, it is difficult to construct one that operates over a broad power envelope down to $\mathcal{O}(100 \mathrm{~W})$ while maintaining relatively high efficiency ${ }^{3}$. Scaling to low power requires a decrease in the thruster channel size and an increase in the magnetic field strength while holding the dimensionless performance scaling parameters constant ${ }^{4,5}$. Increasing the magnetic field becomes technically challenging since the field can more easily saturate the miniaturized inner components of the magnetic circuit and scaling down the magnetic circuit leaves very little room for magnetic pole pieces and heat shields. This makes it difficult to arrive at an optimal magnetic field configuration. Non-optimal fields lead to enhanced power and ion losses which lower efficiency and result in increased heating and erosion of thruster components, particularly the critical inner components comprising the coaxial channel and magnetic circuit. Erosion of the thruster channel is one of the main life-limiting factors in conventional Hall thrusters ${ }^{4}$.

An alternative approach to the miniaturization problem is embodied in the cylindrical Hall thruster $(\mathrm{CHT})^{6,7}$ illustrated in Fig. 1A, which departs from the conventional, purely annular Hall thruster geometry. In contrast to the conventional annular geometry, in the cylindrical geometry the axial potential distribution is critical for electron confinement. This is because there is a large axial gradient in the magnetic field over the cylindrical part of the channel. The electrons drift both azimuthally around the thruster axis and outwards through the $\mu_{e} \nabla B$ force. In the absence of an axial potential, the electrons would mirror out of the region of high magnetic field. In the CHT, the axial potential that accelerates ions outwards also plays an important role in confining the electrons within the thruster by counteracting the mirroring effect. In addition to being unlike an annular Hall thruster, the CHT differs from an end-Hall thruster ${ }^{8}$, which has a purely cylindrical geometry, biased channel walls, and a mostly-axial applied magnetic field. This is in contrast to the cylindrical, ceramic channel with a short, annular region to sustain ionization and produce a 
magnetic field with a strong radial component in the CHT.

The detailed physics in the discharge channel of the CHT has been extensively studied ${ }^{6,7,9}$. In this note we report the measured performance ( $I_{\mathrm{sp}}$, thrust and efficiency) of a cylindrical Hall thruster operating at $\mathcal{O}(100 \mathrm{~W})$ input power.

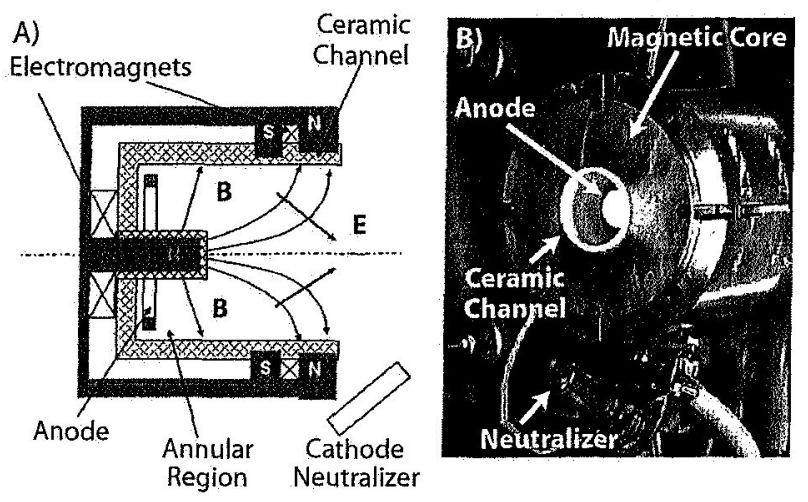

FIG. 1: A) Schematic illustration and B) photograph of the PPPL cylindrical Hall thruster.

\section{EXPERIMENTAL APPARATUS}

Performance measurements are obtained for a $3 \mathrm{~cm}$ cylindrical Hall thruster (see Fig. 1) developed at Princeton Plasma Physics Lab (PPPL). The thruster consists of a boron-nitride ceramic channel, an annular anode, two electromagnet coils, and a magnetic core. The thruster channel is a composite of a shorter, annular region and a longer, cylindrical region. Gas is injected through the anode into the short, annular region of the thruster. The length of the annular region is sized to be greater than the ionization mean free path for xenon. This allows for high ionization of the propellant at the boundary between the annular and cylindrical regions. The electromagnet coils are operated using independently controlled power supplies, and the resulting field topology has a mirror-like structure near the thruster axis.

The working propellant for these experiments is research grade xenon gas. The cathode and anode flowrates are independently controlled using two variable $10 \mathrm{sccm}$ MKS flow controllers (calibrated on Xe and controllable to $\pm 0.1 \mathrm{sccm})$. A commercial HeatWave plasma source is used as the cathode neutralizer. In all experiments, the cathode flowrate is $1 \mathrm{sccm}$.

Thrust was measured using the Variable Amplitude Hanging Pendulum with Extended Range (VAHPER) thrust stand at NASA-Marshall Space Flight Center (MSFC). This thrust stand employs a unique mechanical linkage system that converts horizontal deflection of the pendulum arm into amplified vertical deflection. A 
detailed description of this system is found in Ref. 10. The thrust stand is mounted inside a 9-ft diameter, 25 -ft long stainless steel vacuum chamber. The vacuum level inside the chamber is maintained by two 2400 1/s turbopumps and two $9500 \mathrm{l} / \mathrm{s}$ cryopumps. The base pressure was $5.7 \times 10^{-7}$ Torr and the background pressure of xenon during thruster operation at $\sim 5 \mathrm{sccm}$ total flow rate was $\sim 9 \times 10^{-6}$.

\section{EXPERIMENTAL RESULTS AND DISCUSSION}

Displacement (thrust) calibration of the VAHPER thrust stand is accomplished using an in-situ calibration rig that applies a series of known loads normal to the pendulum arm. Calibration can be performed before, during, and after thruster operation. The measured displacement of the vertically-deflecting linkage is recorded as the calibration loads are applied to the arm. Assuming that the relationship between the applied force and the measured displacement is linear allows for a linear curve fit of the calibration data found in Fig. 2. The calibration routine propagates the errors associated with the individual force and displacement measurements using the uncertainty calculation routine described in Ref. 11.

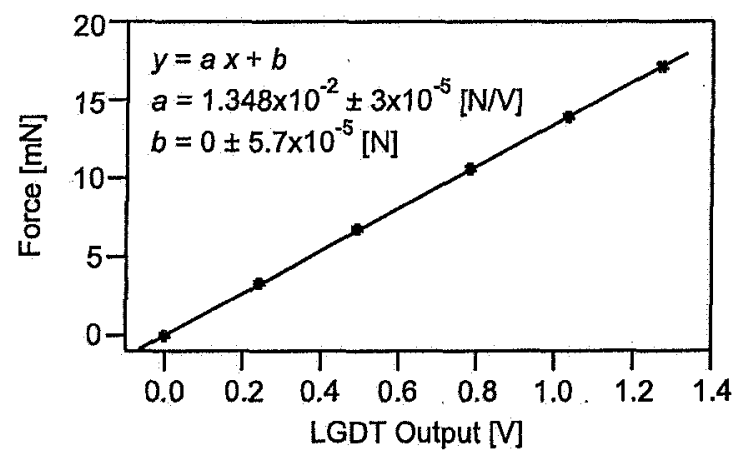

FIG. 2: Applied calibration force plotted as a function of LGDT response. The linear curve is a fit to the displayed data. Error bars on the datapoints and the fit coefficients represent a $68 \%(1 \sigma)$ confidence interval.

Thrust is found by first calculating the Linear Gap Displacement Transducer (LGDT) output voltage difference between the steady-state portion of thruster operation and the zero level output immediately following thruster shutdown (see Fig. 3). These data are then converted to thrust using the calibration curvefit constants found in Fig. 2. Anode efficiency and $I_{\mathrm{sp}}$ are.computed according to their standard definitions 12 using the recorded mass flow rates and power supply outputs. The uncertainty levels on the performance data represent a $95 \%(2 \sigma)$ confidence interval. 


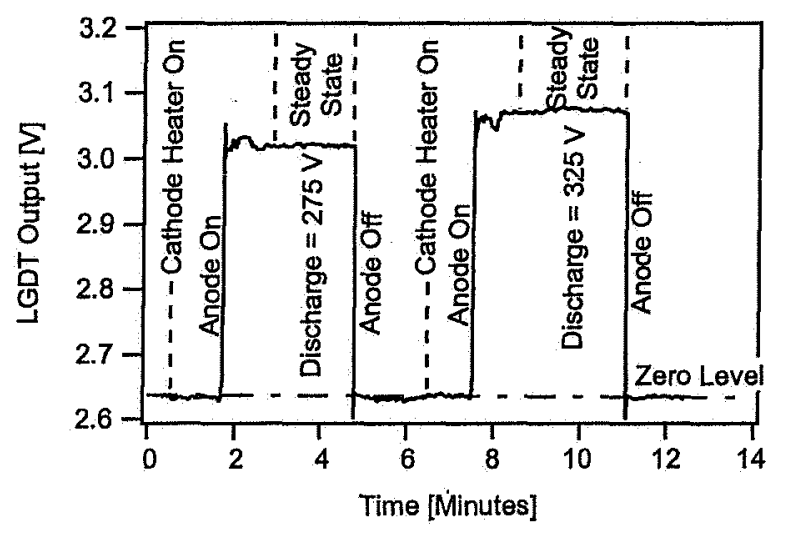

FIG. 3: Raw LGDT output data as a function of elapsed time for two thruster operating points.

Two sets of thrust measurements corresponding to two anode mass flow rates ( 3 and $3.9 \mathrm{sccm}$ ) are presented in Fig. 4A, with the corresponding anode efficiencies plotted in Fig. 4B and $I_{\text {sp }}$ values found in Fig. 4C. These data span a range between 90 to $185 \mathrm{~W}$ in discharge power and result in thrust levels between 3 and $6 \mathrm{mN}$, anode efficiencies between 20 and 27\% and $I_{\mathrm{sp}}$ levels between 1100 and $1650 \mathrm{~s}$. This efficiency compares to the approximately $5 \%$ measured in a miniaturized conventional (coaxial) Hall thruster operating at a power level of $50 \mathrm{~W}^{5}$.

In Fig. 4A, thrust is nearly constant with anode flow rate at a fixed discharge power (note that discharge power is not an independent parameter in a Hall thruster). Anode efficiency (Fig. 4B) increases from 20\% to $27 \%$ with discharge power. In addition, it appears to asymptote at the higher power levels. Specific impulse (Fig. 4C) increases with discharge power and also decreases as the anode flowrate is increased, which is not surprising since total thrust does not vary with anode flowrate.

In Fig. 4, we observe that the lower power data points generally possess much larger errors than the higher power points. The larger errors are partially attributable to recording of insufficient significant figures in the discharge current measurement for those operating points. The issue was resolved before higher power testing, but the error had to be conservatively overestimated for the lower power data set. Additional errors in this lower power set are due to facility-induced drifts in the pendulum arm position, which were greater during that trial than the second, higher power trial. 

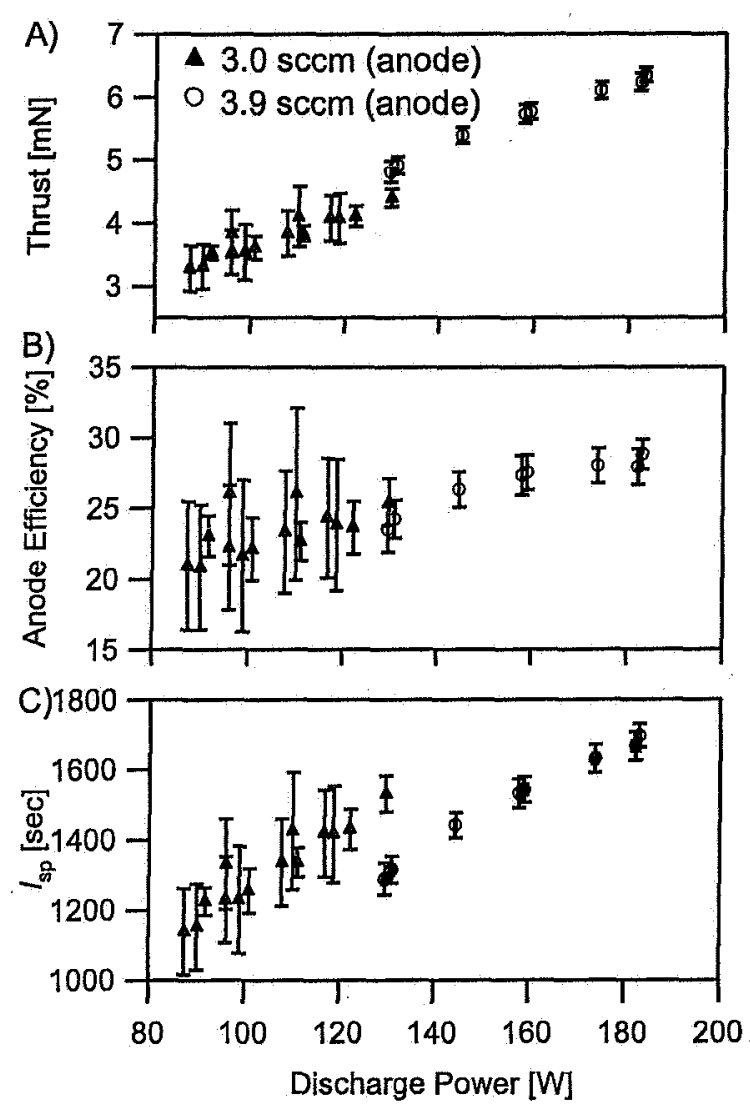

FIG, 4: Performance measurements for a $3 \mathrm{~cm}$ cylindrical Hall thruster: A) Thrust, B) Anode Efficiency, C) $I_{\mathrm{sp}}$ as a function of discharge power. The error bars represent a $95 \%(2 \sigma)$ confidence interval.

\section{CONCLUSIONS}

While conventional (annular) Hall thrusters are efficient in the $\mathrm{kW}$ power regime, they become inefficient when scaled down to small sizes. This is due to the difficulties associated with holding the performance scaling parameters constant while decreasing the channel size and increasing the applied magnetic field strength. The cylindrical Hall thruster can be more readily scaled to smaller sizes due to its non-conventional discharge chamber geometry and associated magnetic field profile.

A series of performance measurements on the PPPL $3 \mathrm{~cm}$ cylindrical Hall thruster over a power ranging from $\sim 85-185 \mathrm{~W}$ were performed using the VAHPER thrust stand. The CHT produced thrust levels ranging from 3-6 mN, anode efficiencies spanning 20-27\%, and $I_{\mathrm{sp}}$ between $1100-1650 \mathrm{~s}$. Thrust increased as a function of discharge power while $I_{s p}$ increased with discharge power and decreased with increasing anode 
flow rate.

\section{Acknowledgments}

We gratefully acknowledge the contributions of the MSFC technical support staff: Doug Davenport, Tommy Reid; Doug Galloway, Keith Chavers and Rondal Boutwell. We also extend our gratitude to students Benjamin Spaun, Chris Dodson and Jeff Gross, and to Freida Lowery in the MSFC Business Development Office. This work performed under Space Act Agreement NAS8-05791 and supported by the MSFC Technology Transfer Office. Work performed by the PPPL co-authors partially supported by the Air Force Office of Scientific Research.

1 Manzella, D., Oh, D.Y., and Aadland, R., "Hall thruster technology for NASA science missions," AIAA Paper 2005-3675, July 2005.

2 Witzberger, K.E. and Manzella, D., "Performance of solar electric powered deep space missions using Hall thruster propulsion," AIAA Paper 2005-4268, July 2005.

3 Mueller, J., "Thruster options for microspacecraft: A review and evaluation of state-of-the-art and emerging technologies," Micropropulsion for Small Spacecraft, edited by M.M. Micci and A.D. Ketsdever, Vol. 187, Progress in Astronautics and Aeronautics, AIAA, Reston, VA, 2000, pp. 45-137.

4 Morozov, A.I. and Savelyev, V.V., "Fundamentals of stationary plasma thurster theory," Reviews of Plasma Physics, edited by B.B. Kadomtsev and V.D. Shafranov, Vol. 21, Consultants Bureau, New York, 2000, pg. 203.

5 Khayms, V. and Martinez-Sanchez, M., "Fifty-Watt Hall thruster for microsatellites," Micropropulsion for Small Spacecraft, edited by M.M. Micci and A.D. Ketsdever, Vol. 187, Progress in Astronautics and Aeronautics, AIAA, Reston, VA, 2000, pp. 233-254.

6 "Raitses, Y. and Fisch, N.J., "Parametric investigations of a nonconventional Hall thruster," Physics of Plasmas, Vol. 8, No. 5, 2001 pp. 2579-2586.

7 Smirnov, A., Raitses, Y., and Fisch, N.J., "Parametric investigations of a miniaturized cylindrical and annular Hall thrusters," Journal of Applied Physics, Vol. 92, No. 10, 2002 pp. 5673-5679.

8 Kaufman, H.R., Robinson, R.S., and Seddon, R.I., "End-Hall ion source," Journal of Vacuum Science and Technology A, Vol. 5, No. 4, 1987 pp. 2081-2084.

9 Smirnov, A., Raitses, Y., and Fisch, N.J., "Plasma measurements in a $100 \mathrm{~W}$ cylindrical Hall thruster," Journal of Applied Physics, Vol. 95, No. 5, 2004 pp. 2283-2292.

10 Polzin, K.A., Markusic, T.E., Stanojev, B.J., DeHoyos, A., and Spaun, B., "Thrust stand for electric propulsion performance evaluation," Review of Scientific Instruments, Accepted for Publication, 2006. 
11 York, D., Evensen, N.M., Martinez, M.L., and Delgado, J.D.B., "Unified equations for the slope, intercept, and standard errors of the best straight line," American Journal of Physics, Vol. 72, No. 3, 2004 pp. 367-375.

12 Jahn, R.G. Physics of Electric Propulsion, McGraw-Hill, New York, 1968, pp. 4, 7. 